\title{
Amplitude-integrated electroencephalography improves the predictive ability of acute bilirubin encephalopathy
}

\author{
Hesheng Chang ${ }^{1 \#}$, Jing Zheng ${ }^{1 \#}$, Jun Ju ${ }^{1}$, Shuxia Huang ${ }^{1}$, Xue Yang ${ }^{1}$, Runyu Tian ${ }^{1}$, Zunjie Liu ${ }^{2}$, \\ Gaifen Liu ${ }^{3}$, Xuanguang Qin ${ }^{1}$ \\ ${ }^{1}$ Department of Pediatrics, Beijing Chaoyang Hospital, Capital Medical University, Beijing, China; ${ }^{2}$ Department of Neonatology, Beijing Obstetrics \\ and Gynecology Hospital, Capital Medical University, Beijing, China; ${ }^{3}$ Department of Neurology, Beijing Tiantan Hospital, Capital Medical \\ University, Beijing, China \\ Contributions: (I) Conception and design: H Chang, J Zheng, G Liu, X Qin; (II) Administrative support: None; (III) Provision of study materials or \\ patients: None; (IV) Collection and assembly of data: H Chang, J Zheng, J Ju, S Huang, X Yang; (V) Data analysis and interpretation: H Chang, J \\ Zheng, J Ju, S Huang, R Tian, Z Liu, G Liu; (VI) Manuscript writing: All authors; (VII) Final approval of manuscript: All authors. \\ "These authors contributed equally to this work. \\ Correspondence to: Xuanguang Qin. Department of Pediatrics, Beijing Chaoyang Hospital, Capital Medical University, Beijing 100020, China. \\ Email: 13801247561@163.com.
}

Background: To establish a clinical prediction model of acute bilirubin encephalopathy (ABE) using amplitude-integrated electroencephalography (aEEG).

Methods: A total of 114 neonatal hyperbilirubinemia patients in the Beijing Chaoyang Hospital from August 2015 to October 2018 were enrolled in this study. There were 62 (54.38\%) males, and the age of patients undergoing aEEG examination was $2-23$ days, with an average of $7.61 \pm 4.08$ days. Participant clinical information, peak bilirubin value, albumin value, hyperbilirubinemia, and the graphic indicators of aEEG were extracted from medical records, and $\mathrm{ABE}$ was diagnosed according to a bilirubin-induced neurological dysfunction (BIND) score $>0$. Multivariable logistic regression was used to establish a clinical prediction model of ABE. Furthermore, decision curve analysis (DCA) was performed to evaluate the model's predictive value.

Results: According to the BIND score, there were a total of 23 (20.18\%) ABE cases. The multivariable logistic regression analysis showed that the value of bilirubin/albumin (B/A), presence of hyperbilirubinemia risk factors, number of sleep-wake cycling (SWC) within 3 hours, widest bandwidth, duration of SWC, and type of SWC were significantly associated with $\mathrm{ABE}$. A clinical prediction model was developed as: $\mathrm{p}=\mathrm{ex} /$ $(1+\mathrm{ex}), \mathrm{X}=0.278+0.713^{*} \mathrm{~B} / \mathrm{A}+2.602 *$ with risk factors (with risk factors equals 1$)-1.500 * \mathrm{SWC}$ number within 3 hours $+0.219^{*}$ the widest bandwidth $-0.065^{*}$ the duration of one SWC $+1.491^{*}$ SWC (mature SWC equals 0 , immature SWC equals 1). The area under the curve (AUC) was 0.85 [95\% confidence interval (CI): 0.750.94], which was significantly higher than the AUC only based on conventional clinical information of B/A (AUC: $0.58,95 \%$ CI: 0.45-0.72). The DCA also showed good predictive ability compared to B/A.

Conclusions: A clinical prediction model can be established based on the patients' B/A, presence of risk factors for hyperbilirubinemia, number of SWC within 3 hours, widest bandwidth, duration of 1 SWC, and the type of SWC. It has good predictive ability and may improve the diagnostic accuracy of ABE.

Keywords: Amplitude integrated electroencephalography; acute bilirubin encephalopathy (ABE); clinical prediction model

Submitted Jan 05, 2021. Accepted for publication Mar 05, 2021.

doi: $10.21037 / \mathrm{tp}-21-35$

View this article at: http://dx.doi.org/10.21037/tp-21-35

^ ORCID: Hesheng Chang, 0000-0003-0983-4653; Jing Zheng, 0000-0002-8362-2125. 


\section{Introduction}

Acute bilirubin encephalopathy (ABE) is among the diseases that seriously endanger the development of nervous system for newborns (1). Its pathogenesis is mainly due to the deposition of bilirubin in the basal ganglia and its toxicity (2). Insufficient understanding of neonatal hyperbilirubinemia may lead to a missed opportunity of early diagnosis of ABE, which can cause severe neurological sequelae $(1,3,4)$, such as abnormal hand and foot movement, auditory impairment, dental dysplasia and intellectual disability. Early detection and identification of bilirubin encephalopathy, and the timely prevention of irreversible brain damage plays a key role in the treatment. The diagnosis of ABE has remained controversial to date (1). At present, bilirubininduced neurological dysfunction (BIND) scoring $(5,6)$, cranial magnetic resonance imaging (MRI) (7) and auditory brainstem response $(1,3,4)$ are the main methods for diagnosing $\mathrm{ABE}$. However, they both have limitations in clinical application. The BIND score is highly subjective and requires long-term observation of patients (4). The high signal of $\mathrm{T} 1$ weighted image (T1WI) in the pallidal region is believed to be a hallmark of ABE (8). However, increased $\mathrm{T} 1$ signal in the pallidal region is also considered a transient phenomenon during the development of myelination, which lacks the specificity of diagnosis $(9,10)$. Auditory brainstem response is also a well-known way of diagnosing ABE, but the relationship of serum bilirubin levels and auditory alterations is still not well defined. Thus the thresholds of blood bilirubin considered critical to the auditory impairment are not determined (11).

Amplitude-integrated electroencephalography (aEEG) is a simplified continuous electroencephalography (EEG) recording method, which has the advantages of simple operation, ease of judging graphics, and long-term bedside operation (12). Findings have proved that aEEG changes can show early than photographic examinations, etc. (13). Numerous data on neonatal brain maturity can be obtained through quantitative reading and scoring of aEEG graphics (14). In recent years, aEEG has been widely used in various fields of neonatology, such as seizure detection $(15,16)$, preterm infants $(17,18)$, metabolic disorder $(19)$, brain damage (20), and hypoxic-ischemic encephalopathy $(21,22)$. However, as a relatively new method of brain function monitoring, whether aEEG could be used to facilitate the early diagnosis of ABE is still unclear.

Our study aimed to develop a clinical prediction model based on aEEG for the early detection of ABE, and evaluate its predictive ability added to conventional clinical information such as bilirubin/albumin (B/A) value.

We present the following article in accordance with the TRIPOD reporting checklist (available at http://dx.doi. org/10.21037/tp-21-35).

\section{Methods}

\section{Participants}

A total of 114 neonates with hyperbilirubinemia admitted to the Beijing Chaoyang Hospital, Capital Medical University, from August 2015 to October 2018 were enrolled in the study. There were $62(54.38 \%)$ males, and the age (in days) of undergoing aEEG examinations ranged from 2 to 23 days, with an average of $7.61 \pm 4.08$ days. Inclusion criteria were as follows: (I) the peak serum total bilirubin level was higher than the $95^{\text {th }}$ percentile of the hourly bilirubin line chart (14); (II) $\geq 35$ weeks of gestation. Exclusion criteria were as follows: (I) presence of congenital craniocerebral dysplasia; (II) presence of neonatal ischemic hypoxic encephalopathy, neonatal respiratory distress syndrome, viral encephalitis, and other diseases affecting the central nervous system; (III) presence of serious genetic metabolic disease.

This study was approved by the Central Ethics Committee at Beijing Chaoyang Hospital, Capital Medical University (the approval No.2019-3-15-2). Written informed consent was signed by legally authorized representatives. All procedures performed in this study involving human participants were in accordance with the Declaration of Helsinki (as revised in 2013).

\section{Clinical data collection}

These clinical data were extracted from medical records: gender, gestational week, weight, age (days) at aEEG examination, feeding (milk feeding, breastfeeding), risk factors for hyperbilirubinemia (with any of the following status: ABO hemolysis, other types of hemolysis, G-6PD deficiency, asphyxia, marked drowsiness, unstable body temperature, sepsis, metabolic acidosis, hypoalbuminemia), peak bilirubin, albumin value, B/A value (i.e., peak B/A value), and BIND Score.

\section{Diagnosis of $A B E$}

The diagnosis of ABE in this study was based on the BIND 
Table 1 The BIND scoring system

\begin{tabular}{llll}
\hline Weight for signs & 1 & 2 & 3 \\
\hline Mental status & Too sleepy; poor feeding & $\begin{array}{l}\text { Very poor feeding, marked lethargy or } \\
\text { irritability }\end{array}$ & Semi-coma; intermittent apnea; seizures \\
Muscle tone & $\begin{array}{l}\text { Slight but persistent } \\
\text { decrease in tone }\end{array}$ & $\begin{array}{l}\text { Moderate hyper or hypotonia depending } \\
\text { on arousal state; posturing, bi-cycling; } \\
\text { mild nuchal and truncal arching }\end{array}$ & $\begin{array}{l}\text { Severe hyper or hyotonia; opisthotonos; } \\
\text { fever }\end{array}$ \\
Cry & High pitched & Shrill and frequent or too frequent & Inconsolable or only with stimulation \\
\hline
\end{tabular}

BIND, bilirubin-induced neurological dysfunction.

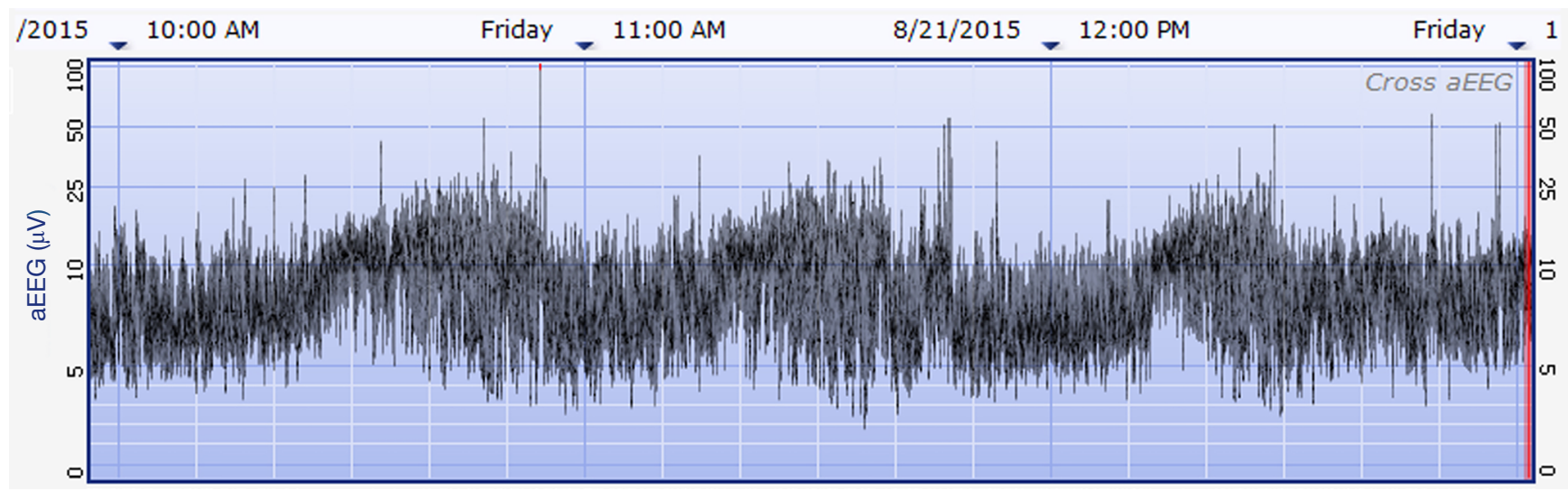

Figure 1 aEEG recording with mature SWC. Both upper and lower boundaries have obvious periodic fluctuations. aEEG, amplitudeintegrated electroencephalography; SWC, sleep-wake cycling.

score, which was scored according to the patient's mental state, muscle tone, and crying (shown in Table 1). Each item was scored 0-3 points, and the total BIND score ranged from 0 to 9 . A BIND score greater than 0 was diagnosed as ABE. The BIND score was evaluated by trained senior pediatric neurologists according to the standard operating procedure.

\section{Amplitude-integrated electroencephalography}

The aEEG was operated and evaluated by trained specialized technicians according to the standard operating procedure with the Olympic Brainz Monitor and Neurofax tracing. The electrodes were placed in bilateral parietal bones. The distance between the 2 points of the electrodes was $75 \mathrm{~mm}$. The midpoint of the 2-point line was $50 \mathrm{~mm}$ posterior to the center of the head, and the reference electrode was placed $25 \mathrm{~mm}$ anterior to the center of the head. The participant was continuously monitored for at least 3 hours. The following parameters were monitored and measured: (I) background continuity: continuous normal voltage was defined as lower boundary voltage $>5 \mu \mathrm{V}$, upper boundary voltage $>10 \mu \mathrm{V}$; discontinuous normal voltage was defined as lower boundary voltage $<5 \mu \mathrm{V}$, upper boundary voltage $>10 \mu \mathrm{V}$. (II) The type of SWC (23): mature indicated that both upper and lower boundaries had obvious periodic fluctuations (Figure 1); immature included unclear (i.e., the lower boundary was visible, but the upper boundary was not obvious or steplike, as shown in Figure 2); easy to be interrupted (i.e., the lower boundary could be seen, but the cycle was poor as shown in Figure 3); none indicated both the upper and lower boundaries were not visible, as shown in Figure 4. (III) The length of one SWC. (IV) The number of SWC within 3 hours. (V) The lower boundary voltage. (VI) The bandwidth: the range between the highest upper boundary voltage and the lowest lower boundary voltage within 3 hours. (VII) The widest bandwidth during quiet sleep. (VIII) The narrowest bandwidth during active sleep. (IX) The proportion of broadband time in one SWC. (X) The 


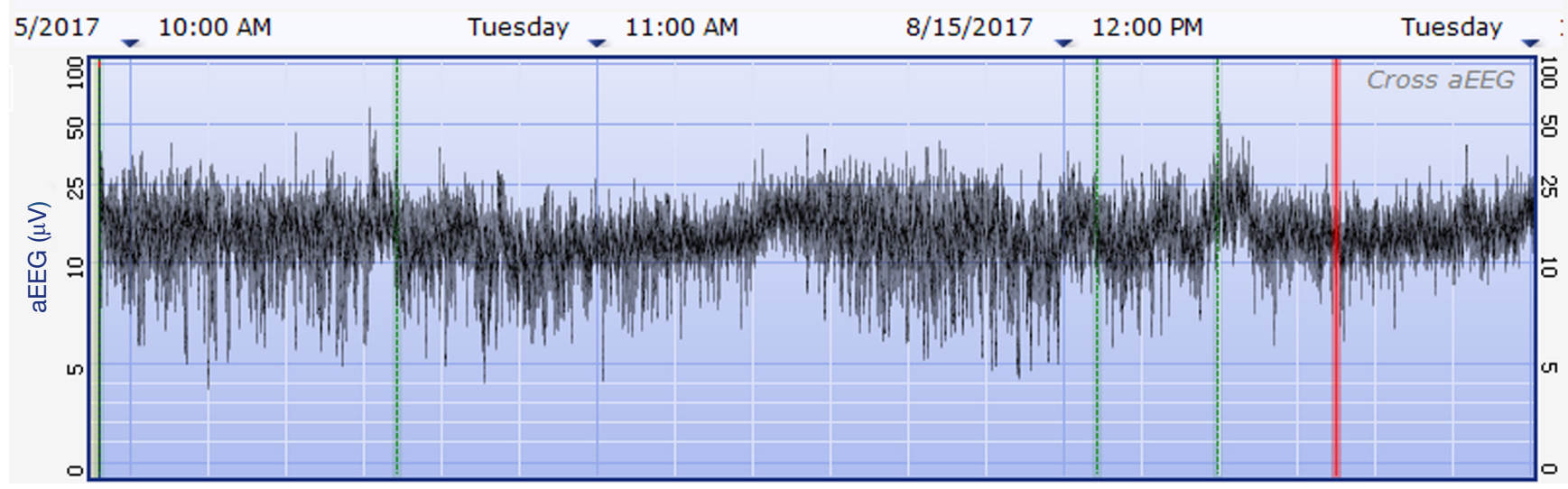

Figure 2 aEEG recording with immature SWC (non-obvious upper boundary). The lower boundary is visible, but the upper boundary is not obvious. aEEG, amplitude-integrated electroencephalography; SWC, sleep-wake cycling.

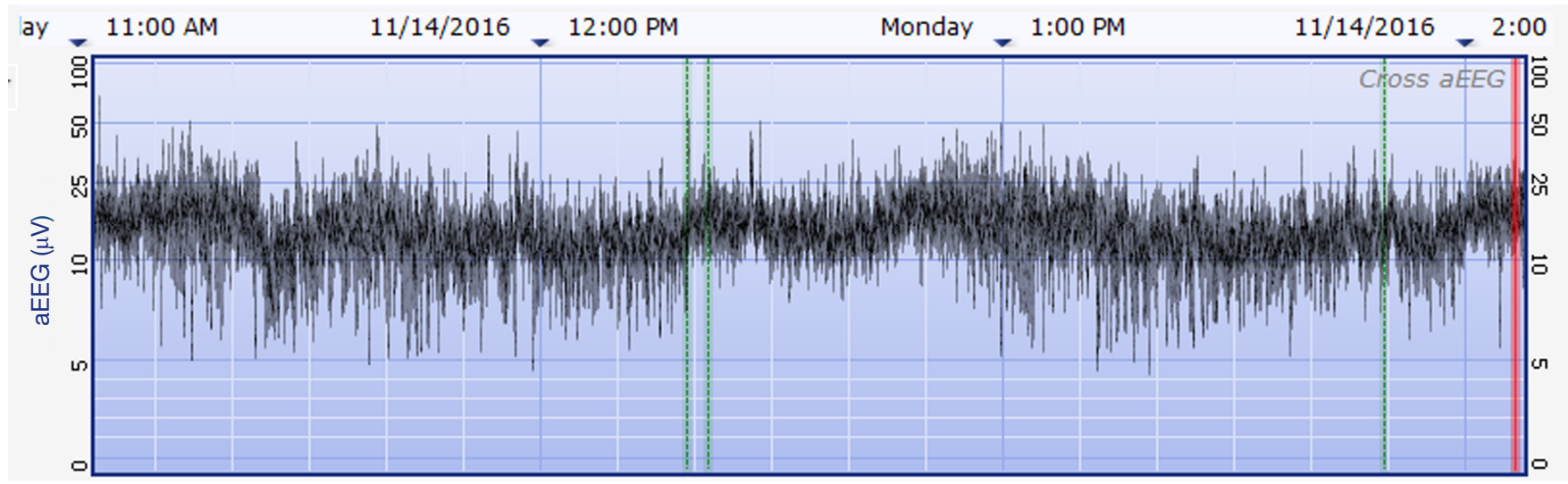

Figure 3 aEEG recording with immature SWC (poor cycles). The lower boundary can be seen, but the SWC cycle is poor. aEEG, amplitude-integrated electroencephalography; SWC, sleep-wake cycling.

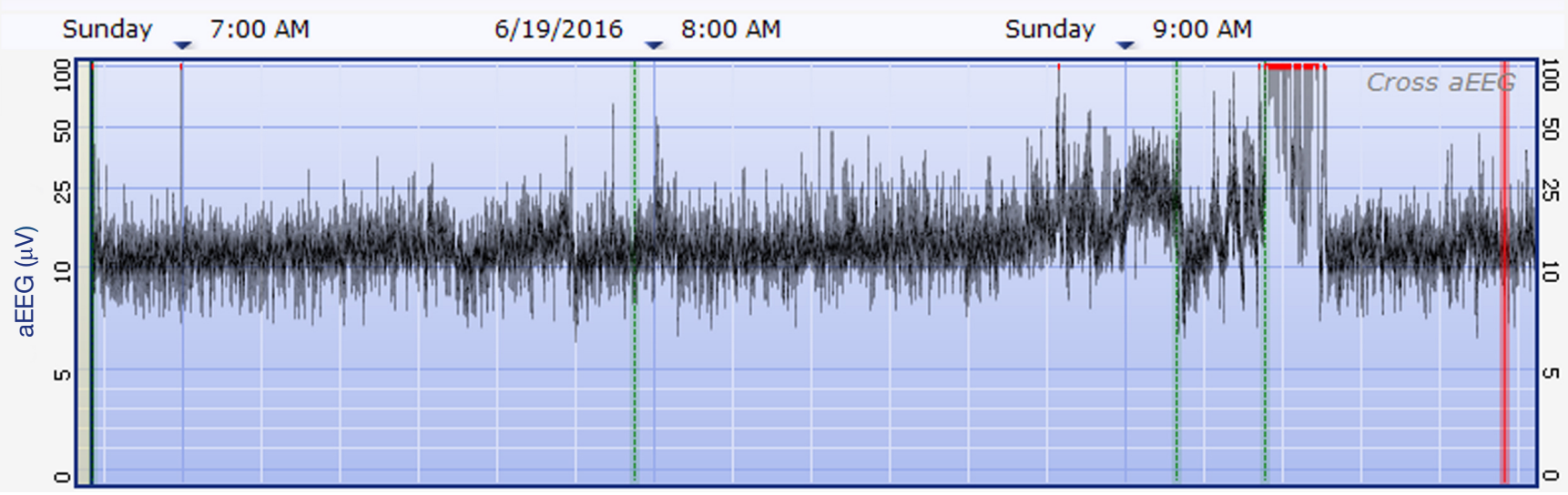

Figure 4 aEEG recording with none SWC. Both the upper and lower boundaries were not visible. aEEG, amplitude-integrated electroencephalography; SWC, sleep-wake cycling. 
Table 2 The clinical characteristics of patients with and without $\mathrm{ABE}$

\begin{tabular}{|c|c|c|c|c|}
\hline Factors & Total $(n=114)$ & $\operatorname{ABE}(n=23)$ & Non-ABE $(n=91)$ & $P$ value \\
\hline Gestational week (day) & $271.89 \pm 9.52$ & $273.26 \pm 9.97$ & $271.55 \pm 9.43$ & 0.44 \\
\hline Weight (g) & $3,300.53 \pm 435.87$ & $3,520.65 \pm 547.63$ & $3,244.89 \pm 386.92$ & 0.006 \\
\hline Age undergoing aEEG (days) & $7.61 \pm 4.08$ & $7 \pm 5.12$ & $7.76 \pm 3.79$ & 0.43 \\
\hline Milk feeding & $55(48.2 \%)$ & $11(47.8 \%)$ & $44(48.4 \%)$ & \\
\hline Breast feeding & $59(51.8 \%)$ & $12(52.2 \%)$ & $47(51.6 \%)$ & \\
\hline With risk factors for hyperbilirubinemia & $9(7.9 \%)$ & $4(17.4 \%)$ & $5(5.5 \%)$ & 0.08 \\
\hline Peak bilirubin value & $362.64 \pm 62.76$ & $379.99 \pm 78.21$ & $358.25 \pm 57.91$ & 0.14 \\
\hline
\end{tabular}

With risk factors for hyperbilirubinemia included anyone of the following status: ABO hemolysis, other types of hemolysis, G-6PD deficiency, asphyxia, marked drowsiness, unstable body temperature, sepsis, metabolic acidosis, hypoalbuminemia. ABE, acute bilirubin encephalopathy; aEEG, amplitude-integrated electroencephalography.

proportion of narrowband time in one SWC.

\section{Statistical analysis}

We used percentage for categorical variables and mean \pm standard deviation (SD) for continuous variables. To compare categorical variables, we used a chi-square $\left(\chi^{2}\right)$ test. For comparing continuous variables, we used the $t$-test or Wilcoxon rank sum test for continuous variables. Multivariate stepwise logistic regression was used to investigate the factors associated with $\mathrm{ABE}$ and establish the clinical prediction model. Area under the curve (AUC) from the C-statistic was used to evaluate the predictive ability. A C-statistic of 1.0 indicated a perfect prediction, whereas a C-statistic of 0.5 indicated that the prediction did not differ from a random prediction. Calibration was assessed using HosmerLemeshow (HL) goodness-of-fit $\chi^{2}$ test. The analysis was performed using the statistical software SPSS 23.0 (IBM Corp., Armonk, NY, USA) and STATA15 (StataCorp LLC. College Station, TX, USA). Finally, the decision curve analysis (DCA) was conducted to evaluate the model using $\mathrm{R}$ 3.6.1 software (https://www.R-project.org/).

\section{Results}

\section{Participant characteristics}

Among the 114 neonates with hyperbilirubinemia, 23 were diagnosed as ABE according to the BIND score, with a prevalence of $20.18 \%$. No significant differences were found among participants regarding gender, age undergoing aEEG, and other factors except weight between the ABE and non-ABE patients (shown in Table 2). The maturation of SWC, duration of 1 SWC, and number of cycles within 3 hours were significantly different between the $\mathrm{ABE}$ and non-ABE participants (Table 3).

\section{Multivariable logistic regression analysis of risk factors for ABE}

Multivariable stepwise logistics regression analysis showed that $\mathrm{B} / \mathrm{A}$, with risk factors for hyperbilirubinemia, the number of SWC within 3 hours, widest bandwidth, duration of 1 SWC, and type of SWC were significantly associated with $\mathrm{ABE}$ (Table 4). The clinical prediction model was finally developed as: $\mathrm{p}=\mathrm{ex} /(1+\mathrm{ex}), \mathrm{X}=0.278+0.713^{*} \mathrm{~B} /$ $\mathrm{A}+2.602^{*}$ with risk factors (with risk factors equals 1 ) $1.500 *$ SWC number within 3 hours $+0.219^{*}$ the widest bandwidth- $0.065^{*}$ the duration of one SWC $+1.491^{*} \mathrm{SWC}$ (mature SWC equals 0, immature SWC equals 1).

\section{Evaluation of clinical prediction model}

The receiver operating characteristic (ROC) curve showed that the AUC was 0.85 [95\% confidence interval (CI): 
Table 3 The aEEG characteristics of patients with and without ABE

\begin{tabular}{lccc}
\hline Factors & Total $(n=114)$ & ABE $(n=23)$ & Non-ABE $(n=91)$ \\
\hline Background continuity & & & P value \\
CNV & $101(88.6 \%)$ & $20(87.0 \%)$ & $0.77(89.0 \%)$ \\
DC & $13(11.4 \%)$ & $3(13.0 \%)$ & $10(11.0 \%)$ \\
SWC & & & $44(48.4 \%)$ \\
Mature & $48(42.1 \%)$ & $4(17.4 \%)$ & $47(51.6 \%)$ \\
Immature & $66(57.9 \%)$ & $19(82.6 \%)$ & $70.88 \pm 15.73$ \\
Duration of one SWC & $68.68 \pm 19.43$ & $60.00 \pm 28.76$ & $2.54 \pm 0.61$ \\
Number of SwC within 3 hours & $2.47 \pm 0.73$ & $2.20 \pm 1.06$ & $5.57 \pm 1.17$ \\
Lower boundary voltage & $5.63 \pm 1.23$ & $5.87 \pm 1.46$ & 0.016 \\
Bandwidth & $24.49 \pm 7.08$ & $27.04 \pm 8.51$ & $23.855 \pm 6.57$ \\
Widest bandwidth & $17.46 \pm 5.04$ & $17.57 \pm 8.67$ & $17.43 \pm 3.67$ \\
Narrowest bandwidth & $7.00 \pm 2.64$ & $7.13 \pm 4.41$ & 0.04 \\
Proportion of broadband time in one SWC & $0.33 \pm 0.11$ & $0.31 \pm 0.17$ & 0.05 \\
\hline
\end{tabular}

aEEG, amplitude-integrated electroencephalography; ABE, acute bilirubin encephalopathy; CNV, continuous normal voltage; DC, discontinuous normal voltage; SWC, sleep-wake cycling.

Table 4 Multivariate stepwise logistic analysis of 114 cases of hyperbilirubinemia

\begin{tabular}{lccc}
\hline & $\beta$ & SE & P value \\
\hline B/A & 0.713 & 0.346 & 0.04 \\
With risk factors for hyperbilirubinemia & 2.602 & 0.959 & 0.007 \\
Number of SWC within 3 hours & -1.500 & 0.728 & 0.04 \\
Widest bandwidth & 0.219 & 0.068 & 0.001 \\
Duration of one SWC & -0.065 & 0.027 & 0.02 \\
SWC & 1.491 & 0.672 & 0.03 \\
\hline
\end{tabular}

$\beta$, adjusted regression coefficient; SE, standard error; B/A, bilirubin/albumin value; SWC, sleep-wake cycling.

0.75-0.94] (Figure 5), and the HL test indicated a $\mathrm{P}$ value of 0.53 . The AUC was significantly higher than the AUC of the model with only the conventional clinical variable of B/A (AUC: 0.58, 95\% CI: 0.45-0.72) ( $\mathrm{P}=0.0003$ ).

The DCA curve showed that the clinical prediction model established has a high clinical prediction value at the threshold of $0.2-1.0$, which had a significant prediction advantage over the B/A model (Figure 6).

\section{Discussion}

This study performed in-depth analysis of the graphic indicators of aEEG, and developed a clinical prediction model of ABE based on aEEG, which significantly improved the predictive ability of $\mathrm{ABE}$ compared to the model based on only the conventional clinical information.

Previous brain function monitoring could only identify the occurrence of brain injury $(18,23)$, but the identification of the different types of brain injury disease often depends on imaging such as cranial nuclear magnetic resonance. However, the accuracy of MRI in diagnosing ABE is still controversial. Since normal myelination can also show different manifestations on images while under developing stage (10), it is difficult to discriminate the differences between diseased and normal myelination (24). Similarly, conventional brain function monitoring for neonates with 
hyperbilirubinemia cannot determine the occurrence of $\mathrm{ABE}$, because it is still unknown which degree of brain dysfunction or kind of specific graphic characteristics are associated with bilirubin encephalopathy. The pathogenesis of $\mathrm{ABE}$ is that bilirubin is deposited in the basal ganglia

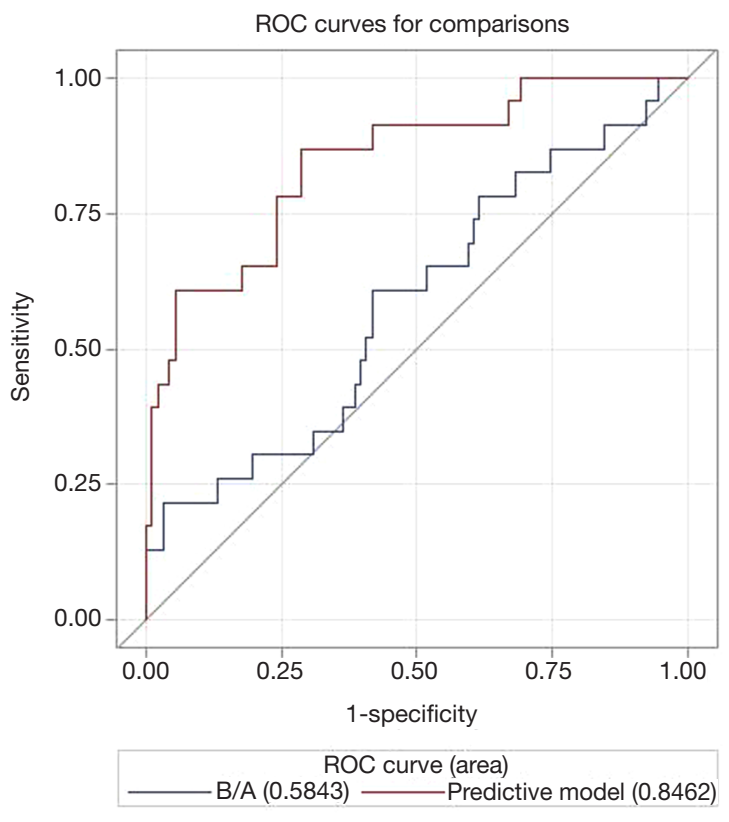

Figure 5 ROC curve comparison between the model of B/A and the model established in the study. The AUC of this clinical prediction model was significantly higher than the AUC of the model with only the conventional clinical variable of B/A. (AUC: 0.85 vs. $0.58, \mathrm{P}=0.0003)$. ROC, receiver operating characteristic.
$(2,25)$, where the aEEG monitoring area (12) locates. Studies in animal models have shown the vulnerable regions of bilirubin encephalopathy were cerebellum, cochlea, oculomotor nucleus, basal ganglia, and hippocampus (25). The hypothalamus globus pallidus loop is closely related to the electrical activity of cortical neurons, and they both participate in the maintenance of the sleep-wake cycle (26). Thus, EEG activity change in the acute phase of bilirubin encephalopathy has its corresponding neuropathological basis. Therefore, we hypothesized that aEEG may play a role in the diagnosis or prediction of ABE; in other words, ABE may have its special graphical characteristics of aEEG.

In our study, we included all known aEEG monitoring indicators in combination with conventional clinical information including $\mathrm{B} / \mathrm{A}$ and risk factors for hyperbilirubinemia. Using multivariable stepwise logistic regression, we developed a clinical predictive model for $\mathrm{ABE}$, which included the following variables: the type of SWC, SWC duration, number of SWC within 3 hours, widest bandwidth, B/A value, and risk factors for hyperbilirubinemia. This clinical predictive model showed an AUC of 0.85 (95\% CI: 0.75-0.94), which was significantly higher than the AUC based only on conventional clinical information of B/A value (AUC: $0.58,95 \%$ CI: $0.45-0.72$ ).

We found that the graphic indicators of SWC and amplitude may be the most sensitive variables, which is in line with findings in previous studies which reported these factors as closely related to central nervous system maturation (14,26-28). Sleep is an important neurological activity in the neonatal period. The observation of neonatal SWC can assist early detection of nerve function injury

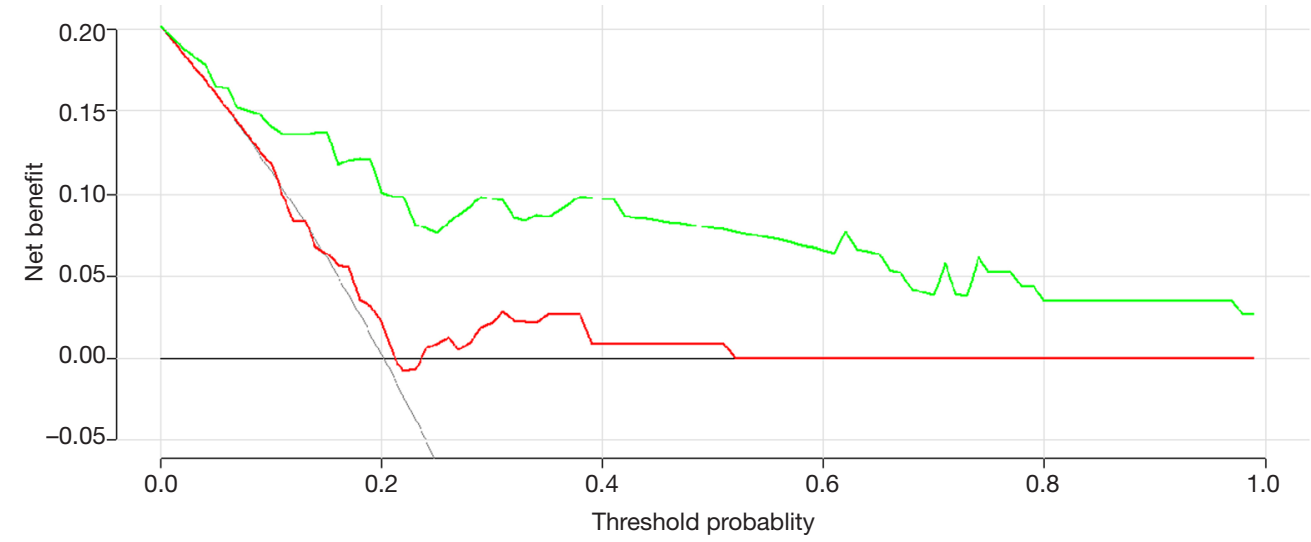

Figure 6 DCA curve. Red line shows the predictive value of B/A, green line shows the predictive value of the prediction model established in this study. This figure shows that the clinical prediction model established has a higher clinical prediction value at the threshold of $0.2-1.0$, $v s$. the B/A model. DCA, decision curve analysis. 
induced by bilirubin, and further analyze the brain function characteristics of ABE. Abnormalities in SWC may be manifested as delayed onset, reduced, or absence of existing SWC. The disappearance or disorder of normal sleep cycle is a serious EEG abnormality, presenting as reductions in both SWC duration and number of SWC within 3 hours. Structure of SWC can also be changed, some studies showed that the quiet sleep period ratio decreased, accordingly the active sleep period ratio increased (29). Our study did not reveal this feature, but showed a pattern of changes with the SWC structure increase of the widest bandwidth. This increase in amplitude is contrary to the brain maturation process, suggesting a correlation with bilirubin toxicity. Previous studies have reported B/A value as a predictor for bilirubin encephalopathy $(29,30)$, with higher total $\mathrm{B} / \mathrm{A}$ ratio increasing the risk of bilirubin encephalopathy by $23 \%$ (odds ratio: $1.23,95 \%$ CI: $1.16-$ 2.48). Our clinical prediction model, which added graphic indicators of aEEG to $\mathrm{B} / \mathrm{A}$ value, significantly improved the predictive ability.

In clinical work, diagnosing neonatal bilirubin encephalopathy quickly and accurately is critical for creating a treatment strategy and prognostic evaluation. Currently, aEEG is the best tool for assessing the brain functional status of newborns (13). It can quickly and accurately assess the degree of brain damage in newborns, but it still lacks specific evaluation indicators. Moreover, it cannot provide evidence which is strong enough to assess the bilirubin encephalopathy based on aEEG alone. The establishment of the clinical prediction model in this study provided a convenient and effective clinical application tool for early identification and judgment of the occurrence of ABE. The AUC of this prediction model was 0.85 , which showed a high predictive value.

Our study had some limitations. In this study, neonates with $\mathrm{ABE}$ treated in our hospital were mainly mild cases and only a few severe cases were included. The predictive ability of this model for neonates with severe ABE needs to be further evaluated, including the effect of seizures and other specific severe aEEG changes Furthermore, this model needs external validation in a multi-center study. In future work, we will perform a multicenter, larger-sample, and prospective cohort study to validate this clinical prediction model, and to evaluate its predictive value for long-term prognosis of neonatal ABE. Survivors of ABE infants will be follow-up and evaluated through multiple measurements, including the magnetic resonance image, auditory brainstem response, Denver Development Screening Test and etc.
Besides, the predictive and diagnosing value of aEEG on preterm or full-term infants as well as hyperbilirubinemia will also be studied in our future studies.

In summary, this study developed a clinical prediction model with good predictive ability for ABE based on aEEG, which is of great significance for the early detection and diagnosis of ABE. However, it needs to be further validated in a future multi-center study.

\section{Acknowledgments}

Funding: The Special Fund of the Pediatric Medical Coordinated Development Center of Beijing Hospitals (XTYB201814).

\section{Footnote}

Reporting Checklist: The authors have completed the TRIPOD reporting checklist. Available at http://dx.doi. org/10.21037/tp-21-35

Data Sharing Statement: Available at http://dx.doi. org/10.21037/tp-21-35

Conflicts of Interest: All authors have completed the ICMJE uniform disclosure form (available at http://dx.doi. org/10.21037/tp-21-35). The authors have no conflicts of interest to declare.

Ethical Statement: The authors are accountable for all aspects of the work in ensuring that questions related to the accuracy or integrity of any part of the work are appropriately investigated and resolved. The study was approved by the central Ethics Committee at Beijing Chaoyang Hospital, Capital Medical University (The approval No.2019-3-15-2). Written informed consent was signed by legally authorized representatives. All procedures performed in this study involving human participants were in accordance with the Declaration of Helsinki (as revised in 2013).

Open Access Statement: This is an Open Access article distributed in accordance with the Creative Commons Attribution-NonCommercial-NoDerivs 4.0 International License (CC BY-NC-ND 4.0), which permits the noncommercial replication and distribution of the article with the strict proviso that no changes or edits are made and the original work is properly cited (including links to both the 
formal publication through the relevant DOI and the license). See: https://creativecommons.org/licenses/by-nc-nd/4.0/.

\section{References}

1. Muchowski KE. Evaluation and treatment of neonatal hyperbilirubinemia. Am Fam Physician 2014;89:873-8.

2. Hachiya Y, Hayashi M. Bilirubin encephalopathy: a study of neuronal subpopulations and neurodegenerative mechanisms in 12 autopsy cases. Brain Dev 2008;30:269-78.

3. Schiavon E, Smalley JL, Newton S, et al. Neuroinflammation and ER-stress are key mechanisms of acute bilirubin toxicity and hearing loss in a mouse model. PLoS One 2018;13:e0201022.

4. Le Pichon JB, Riordan SM, Watchko J, et al. The Neurological Sequelae of Neonatal Hyperbilirubinemia: Definitions, Diagnosis and Treatment of the Kernicterus Spectrum Disorders (KSDs). Curr Pediatr Rev 2017;13:199-209.

5. Radmacher PG, Groves FD, Owa JA, et al. A modified Bilirubin-induced neurologic dysfunction (BIND-M) algorithm is useful in evaluating severity of jaundice in a resource-limited setting. BMC Pediatr 2015;15:28.

6. Olusanya BO, Osibanjo FB, Ajiboye AA, et al. A neurologic dysfunction scoring protocol for jaundiced neonates requiring exchange transfusion. J Matern Fetal Neonatal Med 2018;31:888-94.

7. Liao WH, Wang XY, Wu WL, et al. Differentiation of hypoxic-ischemic encephalopathy and acute bilirubin encephalopathy with magnetic resonance imaging in neonates. Zhongguo Dang Dai Er Ke Za Zhi 2009;11:181-4.

8. Soun JE, Liu MZ, Cauley KA, et al. Evaluation of neonatal brain myelination using the T1- and T2-weighted MRI ratio. J Magn Reson Imaging 2017;46:690-6.

9. Wisnowski JL, Panigrahy A, Painter MJ, et al. Magnetic resonance imaging of bilirubin encephalopathy: current limitations and future promise. Semin Perinatol 2014;38:422-8.

10. Welker KM, Patton A. Assessment of normal myelination with magnetic resonance imaging. Seminars in neurology 2012;32:15-28.

11. Teixeira MH, Borges VMS, Riesgo RDS, et al. Hyperbilirubinemia impact on newborn hearing: a literature review. Rev Assoc Med Bras (1992) 2020;66:1002-8.

12. Shah NA, Wusthoff CJ. How to use: amplitudeintegrated EEG (aEEG). Arch Dis Child Educ Pract Ed
2015;100:75-81.

13. Yuan X, Song J, Gao L, et al. Early Amplitude-Integrated Electroencephalography Predicts Long-Term Outcomes in Term and Near-Term Newborns With Severe Hyperbilirubinemia. Pediatr Neurol 2019;98:68-73.

14. Rhie S, Chae KY, Jo HS, et al. Sleep-wake cycle on amplitude-integrated EEG and neuroimage outcomes in newborns. Ital J Pediatr 2016;42:85.

15. Gacio S. Amplitude-integrated electroencephalography for neonatal seizure detection. An electrophysiological point of view. Arq Neuropsiquiatr 2019;77:122-30.

16. Hellström-Westas L. Amplitude-integrated electroencephalography for seizure detection in newborn infants. Semin Fetal Neonatal Med 2018;23:175-82.

17. Klebermass K, Olischar M, Waldhoer T, et al. Amplitudeintegrated EEG pattern predicts further outcome in preterm infants. Pediatr Res 2011;70:102-8.

18. Benavente-Fernandez I, Lubian-Lopez SP, ZafraRodriguez P, et al. Amplitude-Integrated EEG and Brain Sparing in Preterm Small-for-Gestational-Age Infants. J Clin Neurophysiol 2017;34:456-60.

19. Kadivar M, Mosayebi Z, Badoo RS, et al. Evaluation of the efficacy of amplitude-integrated electroencephalography in the screening of newborns with metabolic disorder admitted to the NICU. BMC Med Imaging 2018;18:33.

20. Variane GFT, Magalhaes M, Gasperine R, et al. Early amplitude-integrated electroencephalography for monitoring neonates at high risk for brain injury. J Pediatr (Rio J) 2017;93:460-6.

21. Goeral K, Urlesberger B, Giordano V, et al. Prediction of Outcome in Neonates with Hypoxic-Ischemic Encephalopathy II: Role of Amplitude-Integrated Electroencephalography and Cerebral Oxygen Saturation Measured by Near-Infrared Spectroscopy. Neonatology 2017;112:193-202.

22. Zhu X, Guo Y, Liu Y, et al. Amplitude-Integrated Electroencephalography for Early Diagnosis and Prognostic Prediction of Hypoxic Encephalopathy in Preterm Infants. Med Sci Monit 2018;24:8795-802.

23. Burdjalov VF, Baumgart S, Spitzer AR. Cerebral function monitoring: a new scoring system for the evaluation of brain maturation in neonates. Pediatrics 2003;112:855-61.

24. Liu Z, Ji B, Zhang Y, et al. Machine Learning Assisted MRI Characterization for Diagnosis of Neonatal Acute Bilirubin Encephalopathy. Front Neurol 2019;10:1018.

25. Wisnowski JL, Panigrahy A, Painter MJ, et al. Magnetic Resonance Imaging Abnormalities in Advanced Acute Bilirubin Encephalopathy Highlight Dentato-Thalamo- 
Cortical Pathways. J Pediatr 2016;174:260-3.

26. Zhang L, Zhou Y, Li X, et al. Hyperbilirubinemia Influences Sleep-Wake Cycles of Term Newborns in a Non-Linear Manner. PLoS One 2017;12:e0169783.

27. Barbeau DY, Weiss MD. Sleep Disturbances in Newborns. Children (Basel) 2017;4:90.

28. Korotchikova I, Stevenson NJ, Livingstone V, et al. Sleep-wake cycle of the healthy term newborn infant in the immediate postnatal period. Clin Neurophysiol 2016;127:2095-101.

Cite this article as: Chang $\mathrm{H}$, Zheng J, Ju J, Huang S, Yang X, Tian R, Liu Z, Liu G, Qin X. Amplitude-integrated electroencephalography improves the predictive ability of acute bilirubin encephalopathy. Transl Pediatr 2021;10(3):647-656. doi: $10.21037 / \mathrm{tp}-21-35$
29. Iskander I, Gamaleldin R, El Houchi S, et al. Serum bilirubin and bilirubin/albumin ratio as predictors of bilirubin encephalopathy. Pediatrics 2014;134:e1330-9.

30. Wang Y, Sheng G, Shi L, et al. Increased serum total bilirubin-albumin ratio was associated with bilirubin encephalopathy in neonates. Biosci Rep 2020;40:BSR20192152.

(English Language Editor: J. Jones) 